\title{
Bronchoscopic diathermy resection and stent insertion: a cost effective treatment for tracheobronchial obstruction
}

\author{
M Petrou, D Kaplan, P Goldstraw
}

\begin{abstract}
Background-Major airways obstruction is a distressing cause of morbidity and mortality. For disease that is extensive and recurrent, there is a need for a safe and cost effective technique for palliation.

Methods-The results of 29 patients with tracheobronchial obstruction (24 malignant and five benign) treated by diathermy resection alone or in combination with endobronchial stenting have been reviewed.

Results-The major site of obstruction was the trachea in 14, main carina in seven, right main bronchus in six, and left main bronchus in two patients. Fifteen had received other forms of treatment beforehand including external radiotherapy, endoscopic dilatation, and laser resection (Nd:YAG). Five patients required two or more treatment sessions for symptom recurrence. Ten patients also received additional treatment with a stent (nine) or insertion of gold grains (one). There were no intraoperative deaths or complications and the average length of stay was five days (range 2-14). Twenty eight patients reported immediate symptomatic relief, and objective improvement in the results of lung function tests was seen in eight patients whose condition was less acute and where preoperative lung function tests could be undertaken (average improvement in $\mathrm{FEV}_{1}$ of $53.1 \%$ and in FVC of 20.6\%).
\end{abstract}

Conclusions-Bronchoscopic diathermy resection is an effective and safe method for relieving the symptoms of tracheobronchial obstruction at appreciably less cost than laser resection.

Department of Thoracic Surgery, Royal Brompton National Heart and Lung Hospital, London SW3 6NP

M Petrou

D Kaplan

P Goldstraw

Reprint requests to: Mr M Petrou

Received 11 March 1993 Returned to authors 28 May 1993

Revised version received

27 July 1993

Accepted 11 August 1993

Tracheobronchial obstruction is a distressing cause of morbidity and mortality in patients with benign and malignant disease. Resection remains the best hope for curative treatment, but is only appropriate for a minority of patients. For the patients who are too frail for surgery and those with benign and malignant disease which is too extensive for resection, there is a need for an effective method of pal- liation. External radiotherapy has become established as the first line treatment for the palliation of malignant airway obstruction. ${ }^{1} \vec{x}$ Endoscopic techniques that have emerged $\stackrel{+}{\infty}$ over recent years include the "core out" method, ${ }^{2}$ afterloading radiotherapy, ${ }^{3}$ cryotherapy, ${ }^{4}$ laser photocoagulation, ${ }^{56}$ and $\vec{G}$ the use of Silastic $^{7}$ and expandable metal stents. ${ }^{89}$ The aim of this study was to show that diathermy resection can be used to relieve airways obstruction safely and effectively.

\section{Methods}

PATIENTS

We have retrospectively reviewed 29 patients who were referred to the Royal Brompton Hospital with tracheobronchial obstruction between November 1985 and April 1992. The average age was 64 years (range 29-84) with 21 men and eight women. Nine patients presented as emergencies with stridor, acute respiratory distress, or both, and 20 were urgent cases complaining of increasing dyspnoea, haemoptysis, or both. Fifteen patients had received previous treatment in the form of external radiotherapy (13), endoscopic $\dot{0}$ dilatation (two) and laser resection (five). One patient had received 22 laser treatment 8 sessions before presenting to us. Tissue for histological examination was obtained from all patients and the pathological findings are $I$ shown in tables 1 and 2 . The major sites of the obstruction are depicted in fig 1 .

\section{MEASUREMENTS}

Response to treatment was assessed subjectively by the patient's account of the symptoms, and objectively by measuring the forced expiratory volume in one second $\left(\mathrm{FEV}_{1}\right)$ and $\cong$ forced vital capacity (FVC) before and after treatment. The poor condition of the patients ${ }_{0}^{\circ}$ prevented objective assessment in over half the cases, and usually these patients were the $\mathbb{\mathscr { D }}$ most dramatically improved by the treatment.

\section{TECHNIQUE}

In all 29 cases general anaesthesia was induced with etomidate at a rate of 0.1 을 $\mathrm{mg} / \mathrm{kg} / \mathrm{min}$ for 10 minutes and maintained by intravenous infusion at a rate of 0.02 $\mathrm{mg} / \mathrm{kg} / \mathrm{min}$. After induction the patients were paralysed with atracurium $0.5 \mathrm{mg} / \mathrm{kg}$ and a Storz 6.5 to 8.5 size adult ventilating rigid 
Table 1 Pathological findings in patients with malignant obstruction

\begin{tabular}{lr}
\hline Malignant tumours & $n$ \\
\hline Primary & \\
$\quad$ Squamous cell carcinoma & 12 \\
Adenoid cystic & 4 \\
& \\
Secondary & 1 \\
$\quad$ Bladder carcinoma & 1 \\
Renal cell carcinoma & 5 \\
Adenocarcinoma of the colon & 1 \\
Giant cell melanoma of the mediastinum & \\
\hline
\end{tabular}

Table 2 Pathological findings in patients with benign obstruction

\begin{tabular}{ll}
\hline Benign conditions & $n$ \\
\hline Neoplastic & \\
Carcinoid & 1 \\
Mucous cell adenoma & 1 \\
Chondroma & 1 \\
Non-neoplastic & \\
Amyloidosis & 1 \\
Fibrous granulation tissue (post-intubation) & 1 \\
\hline
\end{tabular}

bronchoscope was passed into the trachea. Ventilation was then maintained with the Sanders injector. Arterial oxygen saturation was continuously monitored with a pulse oximeter. The diathermy loop (Storz, fig 2) was passed through the bronchoscope and the obstructing lesion resected (fig 3). Debris was removed with biopsy forceps. Once an adequate lumen had been created other options were assessed. Additional treatments were carried out in 10 patients at the first session including the insertion of Silastic stents (eight), a Wallstent (one), and gold grains (one). At the end of the procedure the bronchoscope was removed, an endotracheal tube passed, and mechanical ventilation with nitrous oxide and oxygen used until spontaneous breathing occurred after reversal of neuromuscular blockade with atropine and neostigmine.

Figure 1 Diagram showing the major sites of obstruction for the 29 cases.

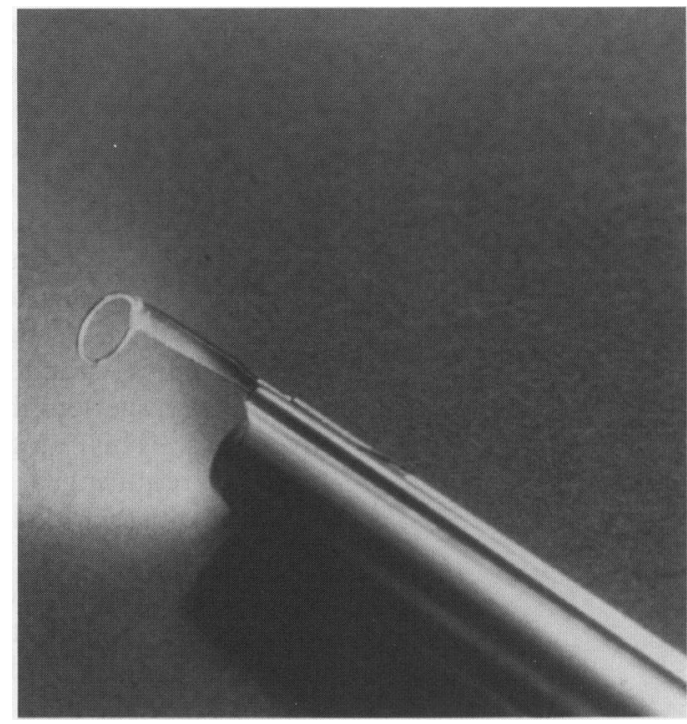

Figure 2 Close-up view of the distal apparatus of the diathermy resectoscope.

\section{Results}

All nine patients requiring urgent diathermy resection obtained immediate and dramatic symptomatic relief. Pretreatment lung function tests could not be performed because of the extreme breathlessness and discomfort experienced by these patients.

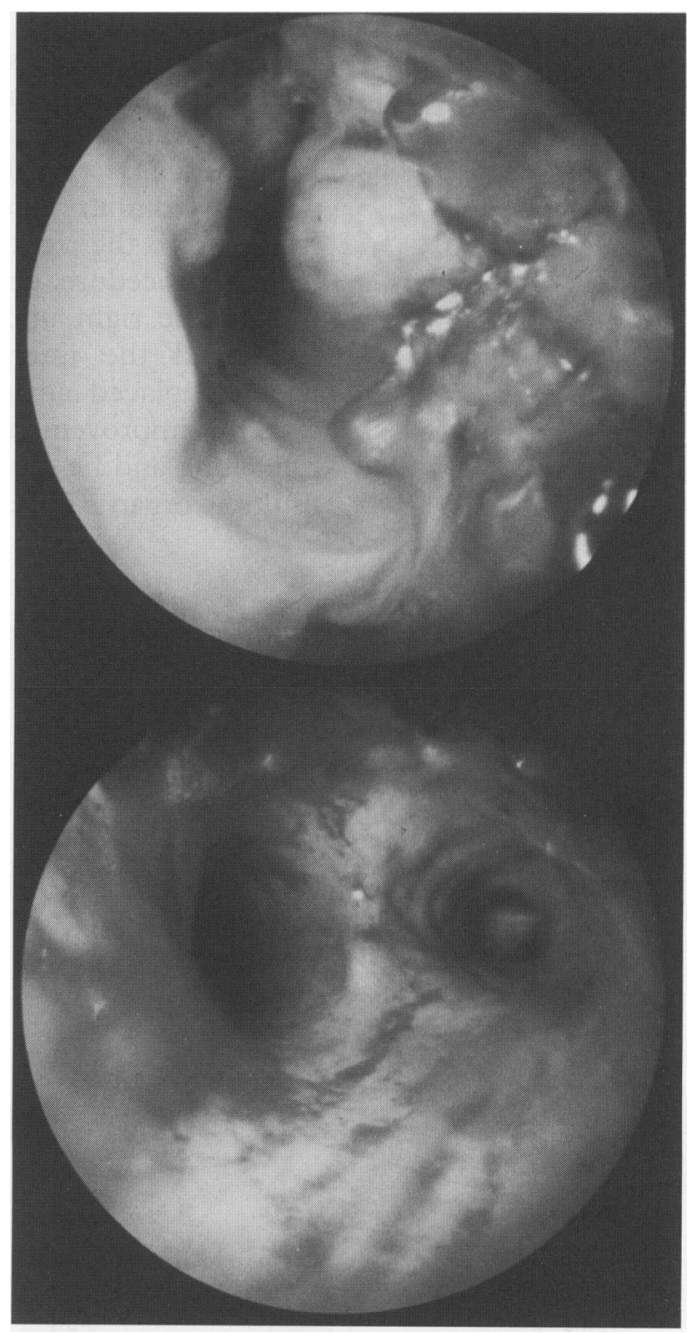

Figure 3 Top: Endoscopic appearance of a patient with critical stridor and undiagnosed tracheal tumour. Bottom: Endoscopic view of the same patient after diathermy resection; stridor was dramatically relieved and samples were obtained for histological examination.
Left main bronchus

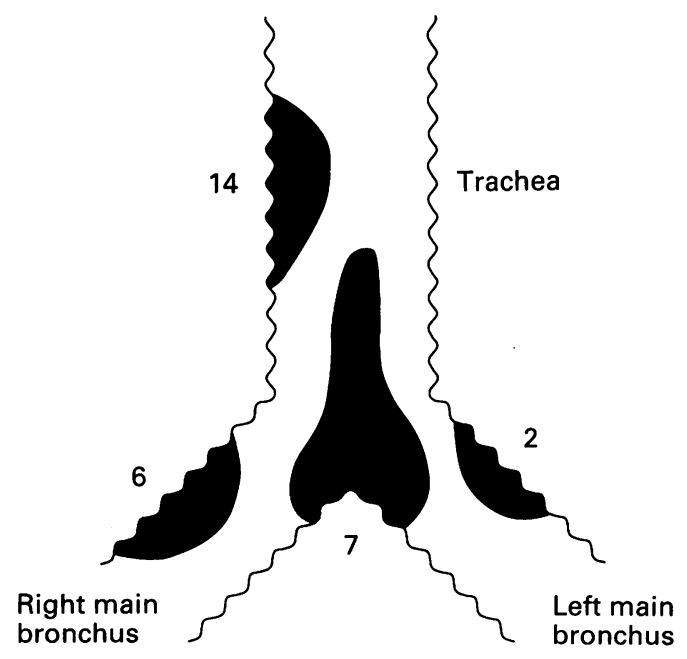

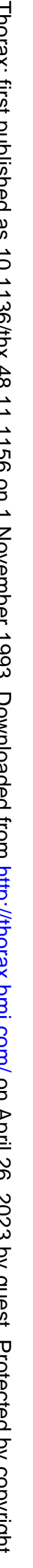


Nineteen of the 20 patients treated electively benefited symptomatically from the treatment, reporting considerable improvement in breathlessness and amelioration of haemoptysis. In all eight patients in whom preoperative lung function tests were performed there was an objective improvement (fig 4). There was a mean improvement in $\mathrm{FEV}_{1}$ of $53.1 \%$ (range $8 \%$ to $142 \%$ ) and in FVC of $20.6 \%$ (range $0 \%$ to $100 \%$ ).

The average length of stay in hospital was five days. This ranged from two days for patients requiring a single treatment session in whom further evaluation was unnecessary, up to 14 days for the 11 patients who went on to receive further treatment in the form of gold grain implantation, afterloading radiotherapy, stenting, and tracheal resection.

There were no intraoperative complications in any patient. Haemorrhage was easily controlled with the diathermy loop and did not compromise the airway. One patient became acutely breathless 12 hours after the operation, associated with a drop in the oxygen saturation from $98 \%$ to $68 \%$. At repeat bronchoscopy an organised clot was seen occluding the left main bronchus which was removed expeditiously and produced an immediate improvement in the oxygen saturation level.

One patient died 24 hours after surgery. He presented with severe dyspnoea due to bilateral multiple lung metastases from a primary adenocarcinoma of the colon. Bronchoscopy revealed a large polypoidal necrotic tumour extending from the lower trachea into the right main bronchus. After a lengthy procedure, despite adequate visualisation of the right upper lobe orifice, tumour still filled the intermediate bronchus and widely replaced adjacent lung tissue. There was no improvement in ventilation of the right lung and he died the following day with multiple organ failure.
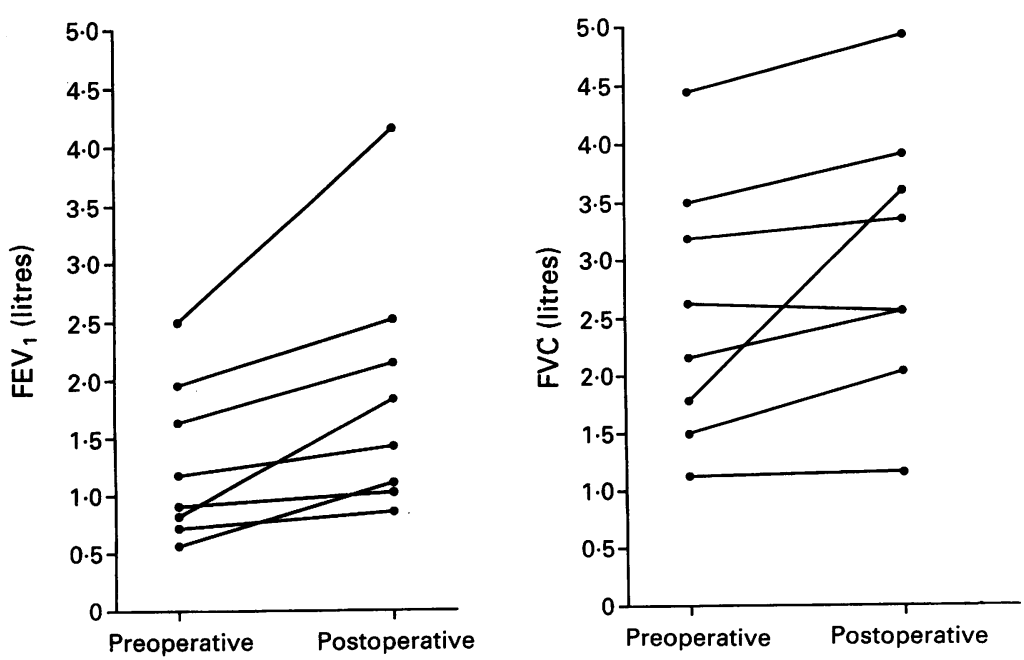

Figure 4 Results of preoperative and postoperative lung function tests.
Follow up at three months was incomplete as these patients are not routinely seen by us in the outpatient department as they are referred back to the physician or oncologist. Five patients had undergone repeat diathermy resection for tumour recurrence without any complications and two patients had died from their underlying malignant disease during this time.

\section{Discussion}

Our experience demonstrates the efficacy and $\vec{O}$ relative safety of using diathermy to resect tracheobronchial obstruction. It is immedi- $\vec{\omega}$ ately available to the endoscopist faced with an emergency airway problem. There is no $x$ systemic toxicity or dosage limit, and is of low $\stackrel{\oplus}{\infty}$

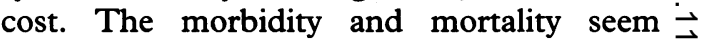
acceptable given the poor state of these patients and the failure of other treatment. $\overrightarrow{\mathrm{S}}$ The one patient who died at 24 hours had 을 advanced disease with total collapse of the lung and the airways completely encased in $z$ tumour tissue. Despite recanalisation, ventilation was not improved. Inpatient stay was short. Elective patients were admitted on the morning of treatment and discharged home the following day.

For patients with malignant strictures, external radiotherapy remains the first line of treatment. ${ }^{1}$ There are, however, problems in treating emergency cases with critical airway obstruction where radiotherapy may precipitate complete obstruction and the consequent logistical problems of an emergency bronchoscopy.

Many endoscopic techniques are available, each having a valuable place in management. Often one uses what options are at hand. The less sophisticated “core out" method, ֻ whereby the bevelled tip of the rigid broncho- 0 scope is used to avulse tumour piecemeal, is associated with a significant risk of haemor- 8 rhage and pneumothorax. ${ }^{2}$ Afterloading radiotherapy $^{3}$ is a recent innovation that 의 requires further evaluation. Cryoresection ${ }^{4}$ is 7 not our first choice as creation of an adequate lumen can be time consuming, often requir- N ing two or more treatment sessions at intervals. In addition, control of bleeding is more $\mathrm{N}$ difficult with the cryoprobe than with diathermy. Since its introduction to clinical practice in 1982, the Nd:YAG laser has become established in the endoscopic man- 0 agement of malignant airways obstruction. ${ }^{56}$ It is, however, an expensive way to undertake $\bar{O}$ a simple mechanical task. The initial outlay is $\mathbb{D}$ f50 000-60 000 and there are continued $\mathbb{D}$ expenses in the upkeep and maintenance of the equipment and the training of staff. Laser photoresection is now performed with the rigid bronchescope under general anaesthesia and shares the logistical problems of all surgical methods. In addition, there are unique risks including fatal and non-fatal haemorrhage ${ }^{1011}$ as well as the potential risk of fires and eye injuries to staff. In contrast, the equipment needed to modulate diathermy 
resection costs our unit $£ 150$, and all other equipment is ready to hand in any surgical endoscopy theatre. Although this series is small, the results are promising and show that it is safe. One important limitation, however, relates to the use of the rigid diathermy probe which does not permit resection of lesions in the upper lobe bronchi. We use the semiflexible grasping forceps (Storz) to remove the tumour piecemeal in this situation, as flexible diathermy probes are currently not generally available.

Diathermy resection for malignant intraluminal tumours provides a repeatable palliative treatment that may be complementary with other therapies - for example, gold grain implantation, ${ }^{12}$ afterloading radiotherapy, and stenting. For benign tumours that are exclusively endolumenal and polypoidal, total resection with diathermy is often possible allowing bronchoscopic treatment.

In non-neoplastic tracheal stenosis diathermy resection is useful for restoring ventilation rapidly, particularly in emergencies. With cases of recurrent tracheal stenosis this techinque affords time for assessment of more definitive therapy such as Silastic $T$ tube/stent insertion or sleeve resection.

We conclude that bronchoscopic diathermy resection provides a cost effective method of palliation in patients with airway obstruction. It is appropriate in non-neoplastic and benign or malignant neoplastic obstruction and is potentially useful as an emergency modality to permit measured assessment before adjuvant treatment.

1 Rostrom AY, Morgan RL. Results of treating primary tumours of the trachea by irradiation. Thorax 1978;33:387-93.

2 Mathisen DJ, Grillo HC. Endoscopic relief of malignant airway obstruction. Ann Thorac Surg 1989;48:469-75.

3 Macha HN, Koch K, Stadler M, Schumacher W, Krumhaar D. New techniques for treating occlusive and stenosing tumours of the trachea and main bronchi: endobronchial irradiation by high dose iridium-192 combined with laser canalisation. Thorax 1987;42: 511-5.

4 Walsh DA, Maiwand MO, Naith AR, Lockwood P, Lloyd MH, Saab M. Bronchoscopic cryotherapy for advanced bronchial carcinoma. Thorax 1990;45:509-13.

5 Hetzel MR, Nixon C, Edmonsone WM, Mitchell DM, Millard FJC, Nanson EM, et al. Laser therapy in a 100 tracheobronchial tumours. Thorax 1985;40:341-5.

6 George PJM, Garrett CPO, Hetzel MR. Role of the Nd:YAG laser in the management of tracheal tumours. Thorax 1987;42:440-4.

7 Tsang V, Goldstraw P. Endobronchial stenting for anastomotic stenosis after sleeve resection. Ann Thorac Surg 1989;48:568-71

8 George PJM, Irving JD, Mantell BS, Rudd RM. Covered expandable metal stent for recurrent tracheal obstruction. Lancet 1990;335:582-4.

9 Tsang V, Williams AM, Goldstraw P. Sequential Silastic and expandable metal stenting for tracheobronchial strictures. Ann Thorac Surg 1992;53:856-60.

10 Cavaliere S, Foccoli P, Farina PL. Nd:YAG laser bronchoscopy. A five year experience in 1000 patients. Chest 1988;94:15-21.

11 Brutinel MW, Cortese DA, McDougall JC, Grillo RG, Bergstrath EJ. A two year experience with the Nd:YAG laser in endobronchial obstruction. Chest 1987;91: 159-65.

12 Ledingham SJM, Goldstraw P. Diathermy resection and radioactive gold grains for palliation of obstruction due to recurrence of bronchial carcinoma after external irradiation. Thorax 1989;44:48-51. 